\title{
PEDIATRIC EWING'S SARCOMA - AN EXPERIENCE IN A TERTIARY CANCER CARE CENTER IN NORTH EASTT INDIA
}

\section{Munlima Hazarika}

Satya S Sarangi*

Bhargab J Saikia

Partha S Roy

Bibhuti Bhusan

Borthakur

Mouchumee Bhattacharyya

\section{Anupam Sarma}

Department of Medical \& Paediatric Oncology, Dr Bhubaneswar Borooah Cancer Institute, Guwahati, Assam, India.

Department of Medical \& Paediatric Oncology, Dr Bhubaneswar Borooah Cancer Institute, Guwahati, Assam, India. *Corresponding Author

Department of Medical \& Paediatric Oncology, Dr Bhubaneswar Borooah Cancer Institute, Guwahati, Assam, India.

Department of Medical \& Paediatric Oncology, Dr Bhubaneswar Borooah Cancer Institute, Guwahati, Assam, India.

Department of Surgery, Dr Bhubaneswar Borooah Cancer Institute, Guwahati, Assam, India.

Department of Radiation Oncology, Dr Bhubaneswar Borooah Cancer Institute, Guwahati, Assam, India.

Department of Pathology, Dr Bhubaneswar Borooah Cancer Institute, Guwahati, Assam, India.

\section{ABSTRACT}

Due to limited clinical data in paediatric Ewing's sarcoma, the aim of this study was to evaluate the demographic characteristics and identifying prognostic factors for survival. We retrospectively reviewed 66 patients with paediatric Ewing's sarcoma. Median age of presentation was 10 years. Male: Female ratio was 1:1.Femur was the most common site of involvement $13.6 \%(9 / 66)$. The median survival in this study was 52 months. Three and five year survival rate of these patients was $52 \%$ and $45 \%$ respectively. One out of the 7 patients ( $14.2 \%$ ) who did not initiate treatment was alive at the time of analysis whereas 4 out of $8(50 \%)$ patients were alive with incomplete treatment and 35 out of 51 patients (68.6\%) were alive who completed treatment and this difference was statistically significant $(p<.001)$.Those patients who completed the treatment protocol had a better survival as compared to those who defaulted or refused treatment.

KEYWORDS : Paediatric, Ewing sarcoma, Demography, Survival patterns

\section{INTRODUCTION}

The Ewing's sarcoma family of tumours (ESFT) is an aggressive form of childhood cancer, which includes classic Ewing's sarcoma, Askin tumour, and peripheral primitive neuroectodermal tumour. This tumor was first described by Ewing in 1921 as "diffuse endothelioma of bone" [1]. The soft tissue counterpart was first reported by Angervall and Enzinger in 1975 [2]. In 1979, Askin et al., reported identical tumors in the thoracopulmonary region which came to be known as Askin tumor.[3] Further work on the molecular characteristics revealed that both ES and PNET shared identical features and these were designated as "ES family of tumors" (ESFT). Before chemotherapy was introduced, about $10 \%$ of patients with Ewing's sarcoma survived [1]. Progress since then has been dramatic, with $75 \%$ of patients with localised tumours now surviving [4]. Progress since then has been dramatic, with $75 \%$ of patients with localised tumours now surviving. However, outcome of patients with metastatic or recurrent disease remains dismal.

\section{MATERIALS AND METHODS}

This retrospective descriptive study includes 66 pediatric patients upto the age of 18 years, treated for Ewing's sarcoma in Dr. B Borooah Cancer Institute, Guwahati, Assam, a grant in aid tertiary cancer care centre of Department of Atomic Energy, Govt. of India, from April 2013 to March 2018. All patients were diagnosed as Ewing's sarcoma on the basis of histopathological examination and immunohistochemistry panels which included CD99, cytokeratin (CK), synaptophysin, chromogranin, NSE, S100, desmin, and LCA. The clinical details were collected from case files. The clinical features such as age, sex, site of involvement, radiological findings, soft tissue extension, metastasis, and recurrence were evaluated.

\section{RESULTS}

This retrospective study comprised 66 children, of whommale and female share equal numbers. Eleven patients belong to the age group 0 to 5 years (16.6\%), 25 from 6 to 10 yrs (37.9\%), 23 from 11 to 15 years $(34.8 \%$ ) and 7 from 16 to 20 years ( $10.6 \%$ ) with a median age of presentation was 10 years. Maximum number of patients were found between 6 to 18 years of age group (48/66, 72.7\%). The most common site of involvement was skeletal involvement in $86.3 \%$ (57/66), followed by soft tissue in $13.6 \%(9 / 66)$. Femur was the most common site of involvement $13.6 \%(9 / 66)$ followed by pelvic bones $10.6 \%$ (7/66), chest wall 6/66 (9\%), scapula and scull bones $7.6 \%$ $(5 / 66)$ and humerus $6 \%(4 / 66) .4$ patients $(6 \%)$ presented with multiple sites involvement.

Among 66 patients 51 completed treatment (77.3\%), 8 patients defaulted (12.1\%) and 7 refused treatment (10.6\%). At the time of analysis [Table no.1], 60.6\% (40/66) patients were alive and $39.4 \%(26 / 66)$ expired.l out of the 7 patients (14.2\%) who did not initiate treatment was alive at the time of analysis where as 4 out of $8(50 \%)$ among patients with incomplete treatment and 35 out of 5 lpatients $(68.6 \%)$ who completed treatment were alive. Among the alive patients $87.5 \%$ completed treatment where as $10 \%$ received incomplete treatment and $2.5 \%$ refused treatment. Forty eight out of 66 patients $(72.7 \%$ ) belong to below poverty line of which 29 were alive at the time of analysis which constituted $72.55 \%$ of the alive patients [Table no 2]. Overall survival at 3years was $52.1 \%$ and 5 years was $45.6 \%$. [Figurel]. There was no significant difference in survival between patients with below and above poverty line $(p=0.981)$. But a significant difference was seen in patients who have completed the planned protocol as compared to those who defaulted/did not underwent treatment. $(\mathrm{p}<$ 0.0001 ). In addition, gender, patient's age, and site of tumour 
Table No.1 : Crosstab Showing Frequency And Precentages Of Different Groups As Per Treatment Completion

\begin{tabular}{|c|c|c|c|c|}
\hline & & \multicolumn{2}{|c|}{ Status } & Total \\
\hline & & Alive & Dead & \\
\hline \multirow[t]{2}{*}{ NO TREATMENT } & Count & 1 & 6 & 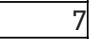 \\
\hline & $\%$ with & $2.5 \%$ & $23.1 \%$ & $10.6 \%$ \\
\hline \multirow{2}{*}{$\begin{array}{l}\text { INCOMPLETE } \\
\text { TREATMENT }\end{array}$} & Count & 4 & 4 & \\
\hline & $\%$ within & $10.0 \%$ & $15.4 \%$ & $12.1 \%$ \\
\hline \multirow{2}{*}{$\begin{array}{l}\text { COMPLETE } \\
\text { TREATMENT }\end{array}$} & Count & 35 & 16 & \\
\hline & $\%$ within & $87.5 \%$ & $61.5 \%$ & $77.3^{\circ}$ \\
\hline \multirow[t]{2}{*}{ Total } & Count & 40 & 26 & 6 \\
\hline & \% within status & $100.0 \%$ & $100.0 \%$ & $100.0^{\circ}$ \\
\hline
\end{tabular}

Table No.2 : Crosstab showing frequency and precentages of different groups as per poverty line status

\begin{tabular}{|l|l|l|r|r|r|}
\hline & & & \multicolumn{2}{|c|}{ Status } & Total \\
\hline & & & Alive & Dead & \\
\hline \multirow{3}{*}{ VAR0002 } & APL & Count & 11 & 7 & 18 \\
\cline { 3 - 6 } & & $\%$ within Status & $27.5 \%$ & $26.9 \%$ & $27.3 \%$ \\
\cline { 2 - 6 } & \multirow{2}{*}{ BPL } & Count & 29 & 19 & 48 \\
\cline { 3 - 6 } & & $\%$ within Status & $72.5 \%$ & $73.1 \%$ & $72.7 \%$ \\
\hline \multirow{2}{*}{ Total } & Count & 40 & 26 & 66 \\
\cline { 2 - 5 } & \% within Status & $100.0 \%$ & $100.0 \%$ & $100.0 \%$ \\
\hline
\end{tabular}

Survival Function

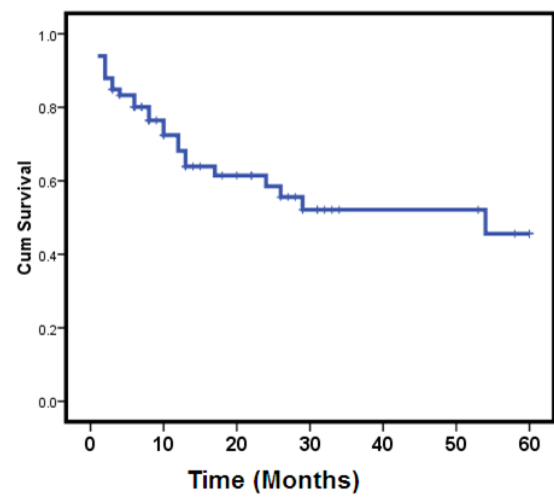

Figure 1: Kaplan Meir curve showing overall survival OVERALL SURVIVAL
1 year

3 Year

5 Year

\section{SURVIVAL RATE SURVIVAL RATE SURVIVAL RATE \\ $68.2 \%$ \\ $52.1 \%$ \\ $45.6 \%$}

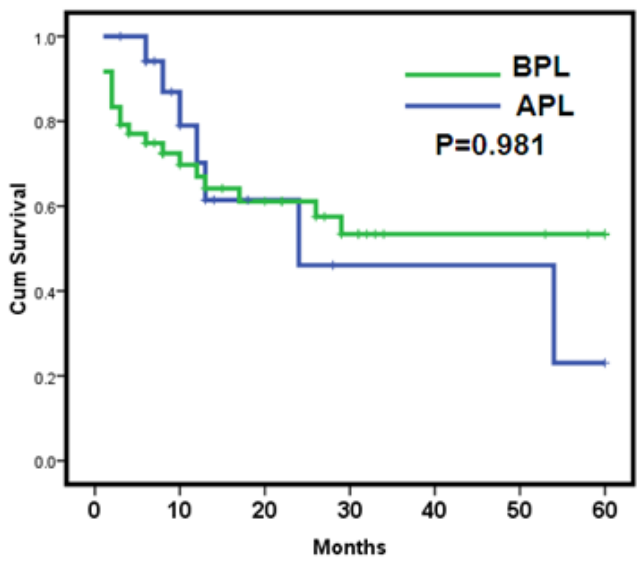

Figure 2: Kaplan Meir Curve Showing Survival According To Economic Condoition

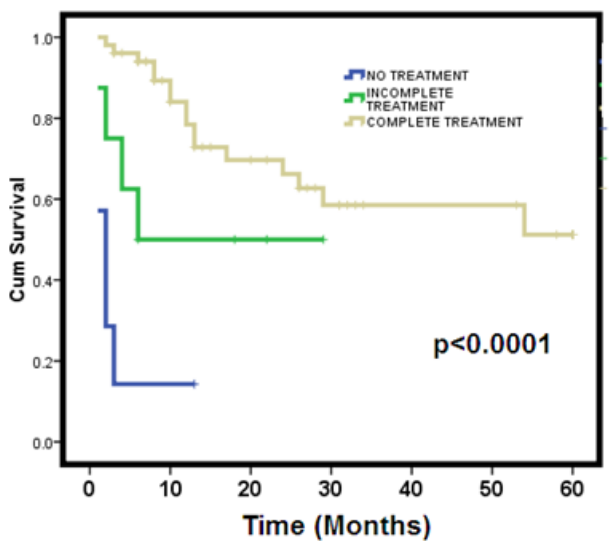

Figure 3: Kaplan Meir Curve Survival According To Treatment competetion Status

\section{DISCUSSION}

In this study, majority of patients were found between 6 to 18 years of age group (48/66, 72.7\%) with a median age of 10 years. Male: Female ratio was 1:1. Femur was the most common site of involvement $13.6 \%$ (9/66). The median survival in this study was 52 months. Three and five year survival rate of these patients was $52 \%$ and $45 \%$ respectively. Close threefourth patients belonged to below poverty line of which $60 \%$ were alive at the time of analysis which constituted $72.55 \%$ of the alive patients. One out of the 7 patients $(14.2 \%)$ who did not initiate treatment was alive at the time of analysis whereas 4 out of $8(50 \%)$ patients were alive with incomplete treatment and 35 out of 51 patients $(68.6 \%)$ were alive who completed treatment and this difference was statistically significant $(\mathrm{p}<.001)$.

The median age reported in our study is in line with previous studies reported in literature [5-6]. Although, there is a male preponderance in many childhood malignancies, but in our study male to female ratio was 1:1. A study by Esmati et al., also showed equal male to female ration in children [7]. The most common site of involvement reported in this study was femur, as previously seen in various studies. [8-9]

Surveillance, Epidemiology, and End Results (SEER) program of the National Cancer Institute have reported five-year survival rates for patients with Ewing sarcoma rose from 36 to 56 percent during the periods 1975 to 1984 and 1985 to 1994 which is also shown by our study with a median survival of 52 months for the entire cohort [10]. There was a significant difference in survival between patients who have completed their treatment vs. who have defaulted or non compliant. Completing the planned chemotherapy regimen had a positive impact on survival while defaulting had detrimental effect on survival as shown by Shanmugam et al. [11] Reason for default being poor compliance among the patients and toxicity associated with chemotherapy.

Socioeconomic status (SES), a multi-dimensional construct that includes economic resources, power and social standing, is associated with a number of health outcomes [12-14]. In our study, there was no difference in outcome between below vs. above poverty line which is in contrast to previous studies. This contrasting result might be because of imbalance in number of patients in both groups.

\section{Strength Of Study}

In the dearth of data for paediatric Ewing's sarcoma patients from India, ours study have provided some database, which to 
the best of our knowledge might be the only study from the north east part of India. This study has tried to highlight the real world scenario for these paediatric patients which are quite different from randomised tricls.

\section{Limitations}

Firstly, this was a retrospective analysis. Secondly, sample size of the study was very less. It might have resulted in non significant $p$ values for many sub group analysis. The compliance rate of treatment was also poor resulting in many patients leaving the treatment protocol midway.

\section{CONCLUSION}

To summarize, this study provides valuable data regarding presentation and management of a rare tumour in paediatric age group from Indian subcontinent. Poor treatment compliance results in worse survival. Thereby, necessitating measures to ensure proper compliance for improved survival, especially in resource constraint settings.

\section{REFERENCES}

1. Ewing J. Diffuse endothelioma of bone. Proc N Y Path Soc 1921:7:17-24.

2. Angervall L, Enzinger FM. Extraskeletal neoplasm resembling Ewing's sarcoma. Cancer 1975;36:240-51.

3. Askin FB, Rosai J, Sibley RK, Dehner LP, McAlister WH. Malignant small cell tumor of the thoracopulmonary region in childhood: A distinctive clinicopathologic entity of uncertain histogenesis. Cancer 1979;43:2438-5.

4. Rosen G, Wollner N, Tan C, et al. Disease-free survival in children with Ewing's sarcoma treated with radiation therapy and adjuvant four-drug sequential chemotherapy. Cancer 1974; 33: 384-93.

5. Glass AG, Fraumeni JF Jr. Epidemiology of bone cancer in children. J Natl Cancer Inst. 1970;44(1):187.

6. Miller RW. Contrasting epidemiology of childhood osteosarcoma, Ewing's tumor, and rhabdomyosarcoma. Natl Cancer Inst Monogr. 1981;(56):9-15.

7. Esmati, E. \& Safaei, A. \& Nosrati, Hassan \& Babaei, Mohammad \& Hashemi, Farnaz \& Farhan, Farshid \& Ashtiani, MS \& Khanjani, Nezhat \& Alibakhshi, Abbas. (2019). Demographic characteristics and prognostic factors in pediatric-type sarcomas; A 7 year single institutional experience and comprehensive review of the current literature. Iranian journal of radiation research (IJRR). 16. 185-195. 10.18869/acadpub.ijrr.16.185.

8. Ginsberg JP, Woo SY, Hicks MJ, Horowitz ME. Ewing's sarcoma family of tumors: Ewing's sarcoma of bone and soft tissue and the peripheral primitive neuroectodermal tumors. In: Priniciples and Practice of Pediatric Oncology, 4th, Pizzo PA, Poplack DG (Eds), Lippincott, Williams and Wilkins, Philadelphia 2002

9. Cotterill SJ, Ahrens S, Paulussen M, et al. Prognostic factors in Ewing's tumor of bone: analysis of 975 patients from the European Intergroup Cooperative Ewing's Sarcoma Study Group. J Clin Oncol. 2000;18(17):3108-3114. doi:10.1200/JCO.2000.18.17.3108.

10. Smith MA, Gurney JG, Ries LA. Cancer in adolescents 15 to 19 years old. In: Cancer incidence and Survival Among Children and Adolescents: United States SEER Program 1975-1995, Ries, LA, Smith, MAS, Gurney, JG, et al (Eds). SEER program, National Cancer Institute, Bethesda MD, 1999.

11. Shanmugam S, Govindasamy G, Hussain S, Fells S. Pediatric bone sarcomas: Outcome of multimodality treatment in a single institution in South India over a decade. Indian Journal of Medical and Paediatric Oncology. 2019;40(5):38.

12. Braveman PA, Cubbin C, Egerter S, Chideya S, Marehi KS, et al. (2005) Socioeconomic status in health research: One size does not fit all. JAMA 294: 2879-2888] [Rabi DM, Edwards AL, Svenson LW, Graham MM, Knudston ML, et al. (2010) Association of median household income with burden of coronary artery disease among individuals with diabetes. Circ Cardiovasc Qual Outcomes 3: 48-53.

13. Frederiksen BL, Osler M, Harling H (2009) Danish Colorectal Cancer Group, Ladelund $\mathrm{S}$, et al (2009) The impact of socioeconomic factors on 30-day mortality following elctive colorectal cancer surgery: $a$ nationwide study. Eur J Cancer 45: 1248-1256].

14. Gupta S, Wilejto M, Pole JD, Guttmann A, Sung L. Low socioeconomic status is associated with worse survival in children with cancer: $\alpha$ systematic review. PLoS One. 2014:9(2):e89482. Published 2014 Feb 26.doi:10.1371/journal. pone.0089482. 